\title{
KM3NeT performance on oscillation and absorption tomography of the Earth
}

\section{Lukas Maderer, ${ }^{a, *}$ João A. B. Coelho ${ }^{a}$ and Véronique Van Elewyck ${ }^{a, c}$ on behalf of the KM3NeT Collaboration}

(a complete list of authors can be found at the end of the proceedings)

and Edouard Kaminski ${ }^{b}$

${ }^{a}$ Université de Paris, APC, CNRS, CEA, Observatoire de Paris, F-75013 Paris, France

${ }^{b}$ Institut Universitaire de France, 1 rue Descartes, Paris, 75005 France

${ }^{c}$ Université de Paris, Institut de physique du globe de Paris, CNRS, F-75005 Paris, France

E-mail: lukas.maderer@apc.in2p3.fr

The KM3NeT neutrino telescope, currently under construction, consists of two detectors in the Mediterranean Sea, ORCA and ARCA, both using arrays of optical modules to detect the Cherenkov light produced by charged particles created in neutrino interactions. Although originally designed for neutrino oscillation and astrophysical research, this experiment also bears unprecedented possibilities for other fields of physics. Here we present its performance for neutrino tomography, i.e. the study of the Earth's internal structure and composition. Owing to the different energy ranges covered by its two detectors ORCA and ARCA, KM3NeT will be the first experiment to perform both oscillation and absorption neutrino tomography. Resonance effects in the oscillations of $\mathrm{GeV}$ neutrinos traversing the Earth will allow KM3NeT/ORCA to measure the electron density along their trajectory, leading to potential constraints of the proton-to-nucleon (Z/A) ratio in the traversed matter. Absorption tomography aims at the detection of neutrinos in the TeV-PeV range with KM3NeT/ARCA. At PeV energies, the Earth is opaque for neutrinos which leads to a reduction of the upgoing neutrino flux at the detector side from which conclusions can be drawn about the density of the inner layers of the Earth. We show here first sensitivity studies of the potential of KM3NeT to address open questions of geophysics concerning the chemical composition and matter distribution in the Earth's core and mantle through neutrino tomography.

$37^{\text {th }}$ International Cosmic Ray Conference (ICRC 2021)

July 12 th - 23rd, 2021

Online - Berlin, Germany

\footnotetext{
*Presenter
} 


\section{Introduction}

The inner structure of the Earth can be simply described as a superposition of different shells of uniform chemical compositions. The planets innermost region is subdivided into a solid core formed of $\mathrm{Fe}-\mathrm{Ni}$, with an approximate radius of $1200 \mathrm{~km}$, surrounded by a molten outer core with a radius of $3480 \mathrm{~km}$ and slightly lower density. Hereafter comes the mantle, in first order uniformly consisting of pyrolite. The outermost layer is a thin $(<100 \mathrm{~km})$ silicate crust. All this knowledge comes from seismic data, geodetic measurements and experimental petrology and led in combination with constraints on radius, total mass and momentum of inertia to the Preliminary Earth Model (PREM) [2]. It is widely used as reference density profile of the Earth and illustrated in Fig. 1.

The upcoming generation of large neutrino telescopes like KM3NeT [4] provide new ways of studying the Earth's interior, mostly independently of seismic measurements. One the one hand, neutrinos with energy larger than few tens $\mathrm{TeV}$ start to get absorbed during their propagation through the Earth. The absorption probability increases with the neutrino energy and the amount of matter along the neutrino path. Energy- and zenith-dependent measurements of the neutrino rates with KM3NeT/ARCA can therefore give insights about the density of the Earth and its layers. This method has been used recently with a one-year sample of muon neutrinos collected with the IceCube neutrino telescope, confirming the feasibility of absorption tomography of the Earth [1]. On the other hand, KM3NeT/ORCA will detect $\sim \mathrm{GeV}$ neutrinos whose mean free path is much bigger than the Earth's diameter. Instead of being absorbed, those lower energetic neutrinos "feel" the presence of matter, i.e electrons, as an extra potential to their propagation Hamiltonian. This leads to resonance effects in their oscillation probability, which depends on the electron density $n_{e}$ within the traversed matter, where

$$
n_{e}=\frac{N_{A}}{m_{n}} \times \frac{Z}{A} \times \rho_{m}
$$

with the Avogadro number $N_{A}$ and the nucleon mass $m_{n}$. The effective proton-to-nucleon ratio $Z / A$ varies among chemical elements, e.g. it is 0.4655 for $\mathrm{Fe}$ and 1 for $\mathrm{H}$. It can therefore be used to constrain the nature and abundances of light elements in the outer core, which can not be revealed by seismologic measurements only, but whose presence is required to explain the jump in density between inner and outer core [3]. Constraining the inner Earth composition will also improve our understanding of the dominant processes that led to the formation of our planet. Our analysis combines models for the neutrino flux, and cross section, and for the inner structure of the Earth to compute the number of events at the detector site. The neutrino oscillation or absorption probabilities are affected by the Earth parameters, i.e. $Z / A$ and $\rho_{m}$. The detector response is simulated using a response matrix calculated with MC simulations (discussed in Sec.2). We find that after 10 years of data taking with two KM3NeT/ARCA building blocks, we will be able to profile the Earth density with comparable sensitivity as a similar study by IceCube [1]. With 10 years of KM3NeT/ORCA data we can set limits on the outer cores $Z / A$ and density (see Sec.3). 


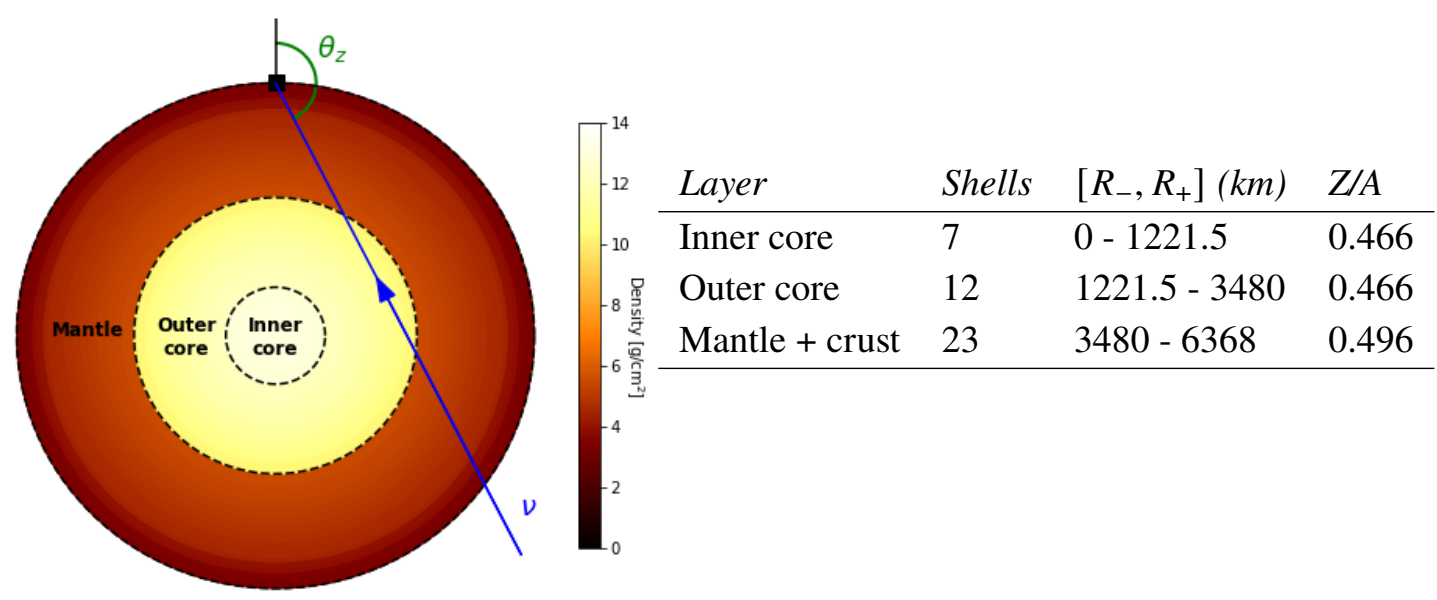

Figure 1: Left: Terrestrial density profile according to the 42-shells model used in this contribution, also showing the main inner compositional layers. Right: Compositional layers with the associated number of constant density shells, the exact innermost and outermost radius, and the corresponding default $\mathrm{Z} / \mathrm{A}$ value $[5,6]$.

\section{Methodology}

\subsection{Underlying signal}

The input model of the atmospheric neutrino flux is taken from Honda $(2015)^{1}$ [7] for energies $1-100 \mathrm{GeV}$. Between $100 \mathrm{GeV}$ and $100 \mathrm{PeV}$ we use a combination of the conventional flux taken from Honda(2007) [8] and a prompt neutrino flux from Enberg [9]. To simulate the effects of matter on neutrinos traversing the Earth, for both oscillation and absorption, the OscProb software [10] is used.

For a given incident angle $\theta_{z}$, the trajectory of a neutrino through the Earth is modelled along the corresponding baseline with a sequence of steps of constant matter and $Z / A$ and thus the electron density (according to Eq. 1). The latter induces an effective potential for charged-current interactions $V_{C C}= \pm \sqrt{2} G_{F} N_{e}(x)$ (where \pm applies respectively to $v_{e}$ and $\bar{v}_{e}$ ), which modifies the neutrino oscillation probabilities. These are calculated from the quantum evolution equations for the neutrino states for each shell along the neutrino path. The absorption probability within each shell is calculated using the simple exponential function

$$
P_{\mathrm{Abs}}(E, \Theta)=\Pi_{i} e^{-l_{i}(\theta) \cdot \frac{\rho_{i}}{u} \cdot \sigma(E)}
$$

with $l$ the path length through the respective shell $i, \rho$ its density, $u$ the atomic mass number and $\sigma$ the neutrino-nucleon cross-section weighted for water molecules, obtained with the GENIE Monte Carlo generator [11].

The latter is also used to compute the rate of events interacting in the detector. This differential rate (corresponding to the number of neutrino interactions of given flavour, energy $E$ and zenith $\theta$ occurring in the detector per unit exposure ${ }^{2}$ ) is computed for each (anti-)neutrino flavour $\beta$ as:

$$
\frac{\mathrm{d} N_{\beta}^{\mathrm{int}}(E, \theta)}{\mathrm{d} E \mathrm{~d} \theta}=\sigma_{v_{\beta}}(E) \cdot \sum_{v_{\alpha}} P_{v_{\alpha} \rightarrow v_{\beta}}(E, \theta) \cdot\left(1-P_{A b s, \beta}(E, \theta)\right) \cdot \frac{\mathrm{d} \Phi_{v_{\alpha}}}{\mathrm{d} E \mathrm{~d} \theta}(E, \theta)
$$

${ }^{1}$ We have used the tables for Gran Sasso site without mountain, azimuth-averaged, at solar minimum.

${ }^{2}$ The term exposure corresponds to the product of the detector lifetime and its effective mass [Mton years] 

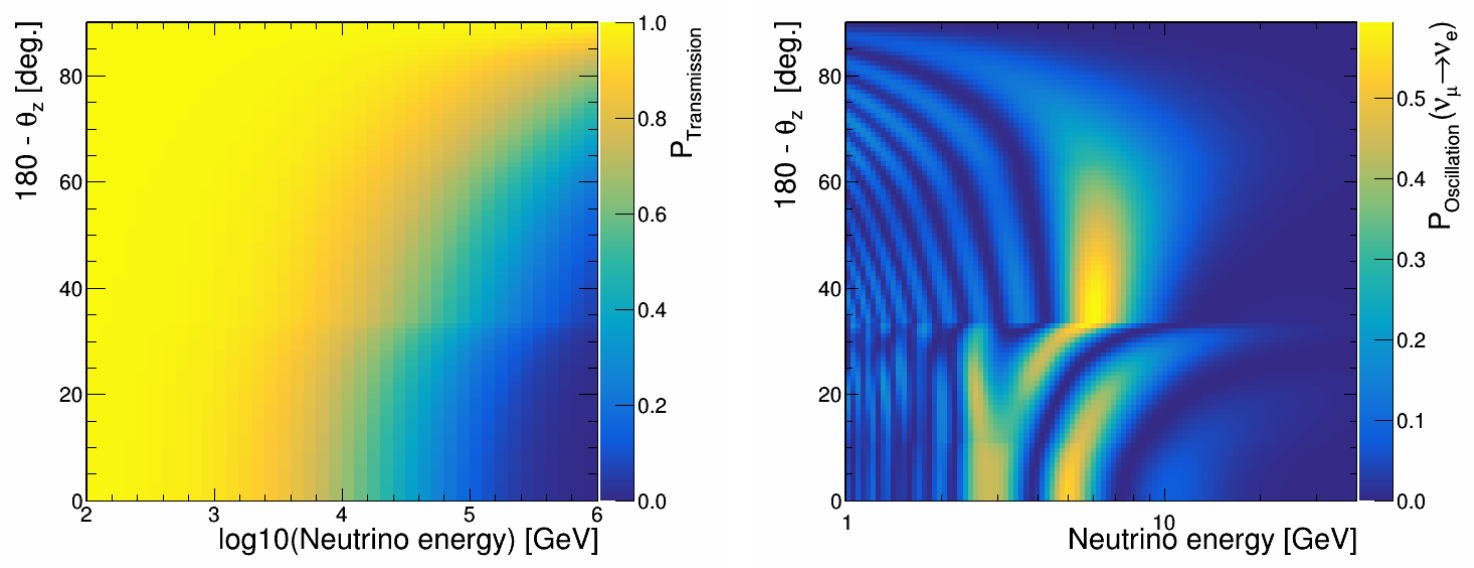

Figure 2: Left: Transmission probability for $v_{\mu}$; Right: Oscillation probability for $v_{e} \rightarrow v_{\mu}$ transition. Both histograms are computed with OscProb [10]. The y-axis ranges from up-going to horizontal neutrino events.

where $\sigma_{v_{\beta}}$ is the total interaction cross-section for neutrino type $v_{\beta}, P_{\alpha \rightarrow \beta}$ is the $v_{\alpha} \rightarrow v_{\beta}$ oscillation probability, and $\mathrm{d} \Phi_{v_{\alpha}} / \mathrm{d} E \mathrm{~d} \theta$ is the unoscillated differential flux of atmospheric neutrinos at the detector location. For studies with ARCA we neglect oscillation effects, i.e. $P_{\alpha \rightarrow \beta}=\delta_{\alpha \beta}$, for studies with ORCA we neglect absorption effects, i.e. $P_{\text {Abs }}=0$.

\subsection{Detector response modelling}

The KM3NeT collaboration is currently building a network of water Cherenkov neutrino telescopes in the Mediterranean sea. The two detectors ORCA and ARCA (Oscillation/Astrophysical research of cosmics in the abyss[4]) are using the same technical components, 3D arrays of digital optical modules (DOM) hosting 31 small photomultipliers each to detect Cherenkov light from particle showers of neutrino-nuclei interactions, distributed along slender strings, anchored at the sea bottom at a depth of about 2500 and $3500 \mathrm{~m}$ respectively. The KM3NeT DOMs detect the Cherenkov light induced by charged particles that are generated in the interaction of a neutrino with the matter surrounding the array. The detector layout, i.e. horizontal and vertical spacing of the DOMs, determines the threshold energy that is needed to trigger an event in the detector. KM3NeT/ORCA, with an instrumented volume equivalent to $\sim 7 \mathrm{Mtons}$, will cover the $1-100 \mathrm{GeV}$ energy range, while KM3NeT/ARCA, with $\sim 1$ Gton fiducial mass, detects neutrinos at higher energies. One distinguishes between two event topologies, track- and shower-like. A $v_{\mu}$ that interact with a nucleus via a charged current (CC) create a $\mu$ that will propagate through the detector, and inducing the emission of Cherenkov light along its trajectory.All $v_{e}$ and neutral-current (NC) interactions induce an approximately spherical light emission around the interaction point and are thus referred to as shower-like events. A special case are $v_{\tau}$-CC, where the produced $\tau$-lepton can either decay into a $e$ or a $\mu$. Note that we did not simulate $v_{\tau}$-events for ARCA, because their total contribution is negligible (only produced by charmed meson decays).

Different approaches to modelling the KM3NeT detector response have been used so far in the literature. The analysis presented in Bourret (2019) [12] used parametrized response 
functions that allowed to perform sensitivity studies for different detector types. In the present work, we use instead the same approach as in Bourret (2017), based on a full event-by-event Monte Carlo simulation that repoduces more accurately the specific response of the KM3NeT detectors [13]. Based on these simulations, we create a reponse matrix $\mathrm{R}$ with a binning in five dimensions $\left(E_{\text {True }}, \theta_{\text {True }}, E_{\text {Reco }}, \theta_{\text {Reco }}, f\right.$ ) where $f$ encrypts the interaction channel and reconstructed class (trackor shower-like). For a given event with true characteristics $E_{\text {True }}, \theta_{\text {True }}$, the response function R provides the probability for this event to be classified in a specific class and ( $\left.E_{\text {Reco }}, \theta_{\text {Reco }}\right)$ bin.

\subsection{Statistical methods}

With the methods described above we can now simulate experiments based on given input models, in our case changing density and $Z / A$ values for different layers of the Earth. We compute then the log-likelihood ratio for two Asimov datasets, i.e. pseudo-experiments where the data corresponds to the mean of the expected results given a set of input parameters. Assuming that the number of events per bin follows Poisson statistics, one can define

$$
\chi_{\text {hyp }}^{2}(\text { data })=\sum_{b=1}^{\text {Nbins }} 2\left[\left(\mu_{\mathrm{hyp}}\right)_{b}-n_{b}+n_{b} \ln \left(\frac{n_{b}}{\left(\mu_{\mathrm{hyp}}\right)_{b}}\right)\right]
$$

which corresponds to the likelihood to measure the simulated data with an underlying true hypothesis hyp. The resulting Asimov sensitivity $S_{\text {Asimov }}=\sqrt{\left|\Delta \chi^{2}\right|}$ is in good agreement with the sensitivity obtained from the log likelihood ratio (with some caveats (see [13]), where

$$
\Delta \chi^{2}=\chi_{\text {prem }}^{2}\left(\text { data }_{\mathrm{prem}}\right)-\chi_{\text {hyp }}^{2}\left(d a t a_{\mathrm{prem}}\right)=-\chi_{\text {hyp }}^{2}\left(\text { data }_{\mathrm{prem}}\right) .
$$

In this case $S_{\text {Asimov }}$ corresponds to the confidence level to which the hypothetical Earth model can be distinguished from PREM.

\section{Results and discussion}

\subsection{Absorption tomography}

Analogously to the analysis reported in [1], performed with 1-year data of IceCube, we use an Earth model with 5 layers with radii of [1242, 2372, 3502, 4935, 6368](km), such that we have one layer for the inner core and two layers for outer core and mantle, respectively.

We fit simultaneously the densities of the 5 layers to our simulated data and determine the $1 \sigma$ uncertainties with ROOT::Minuit2 [14] without taking into account any systematic uncertainty.

The result shown in Fig. 3 is comparable with the prediction for 10 years of IceCube data presented in [1] (note that our first bin [0-1242 km] appears to be larger due to the logarithmic $y$-scale). The width of the error bands decreases with the total volume of the respective layer. While only a fraction of the detected neutrinos traversed the inner core, all of them pass through the mantle and thus the statistical uncertainty for the density fit of the mantle is reduced. Although finer features of the Earth's density profile like the density step between inner and outer core can not be resolved, ARCA is able to distinguish the core from the mantle region. 


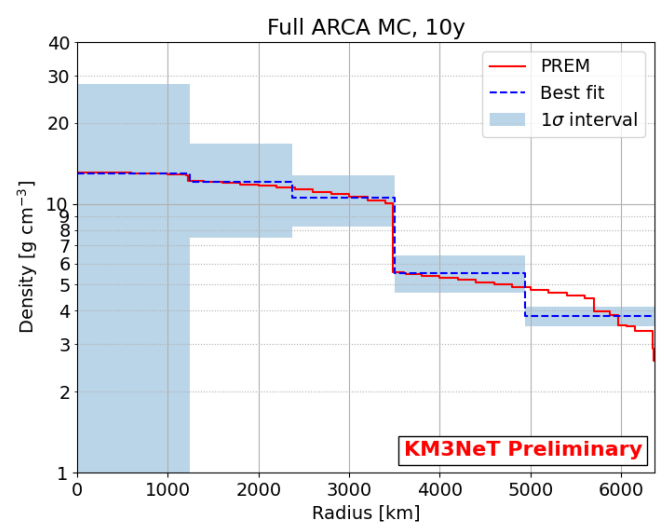

Figure 3: 5-layer fit of the Earth density and $68 \%$ uncertainties for a simulated lifetime 10 years for both building blocks of KM3NeT/ARCA. No systematic effects are included. The red curve shows the underlying 42-layer PREM density profile used for the simulation of the experiment.
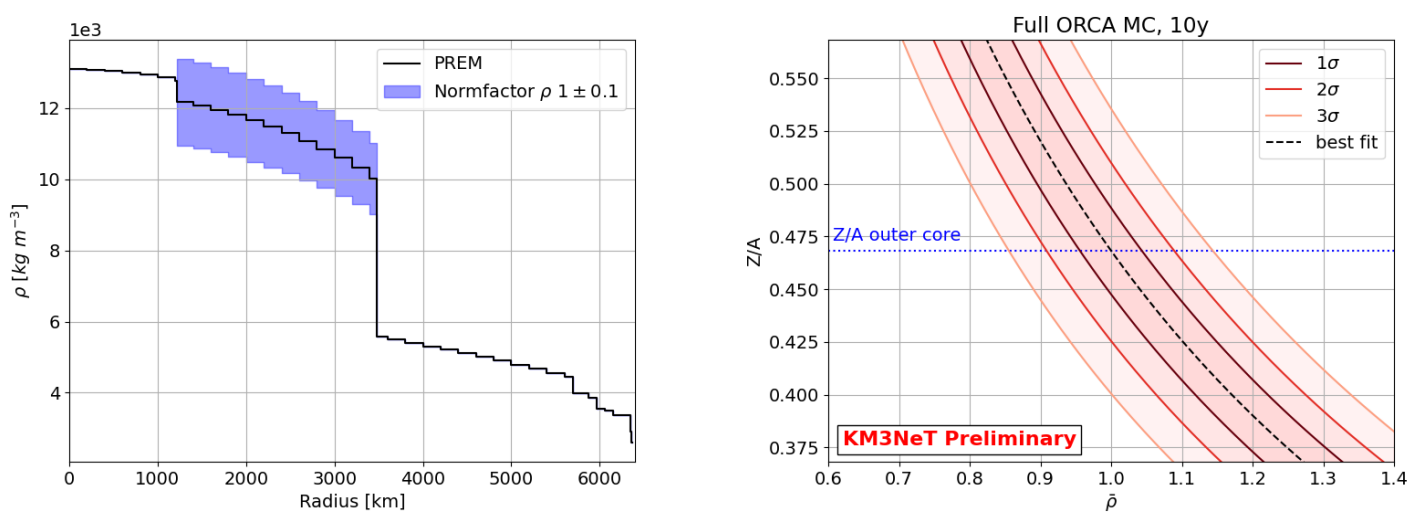

Figure 4: Left:Visualisation of $\bar{\rho}$. We use it to scale the density of all 12 model layers of the outer core simultaneously. Right: Sensitivity contours for $Z / A$ and $\bar{\rho}$. The error intervals are calculated for $\bar{\rho}=1$, the contour lines correspond to the combinations of $Z / A$ and $\rho$ that result in the same $n_{e}$.

\subsection{Oscillation tomography}

Our goal for the oscillation tomography is to constrain the electron density $n_{e}$ in the outer core and find hints on its chemical composition. Because $n_{e}$ is the direct product of the matter density $\rho_{m}$ and the proton-to-nucleon ratio $Z / A$ (see Eq. 1), a fit of $n_{e}$ is analogous to either a fit of the $Z / A$ value or a fit of a normalisation factor $\bar{\rho}$ for the density as illustrated in Fig. 4 (left), while keeping the respective other parameter constant.We fit the $Z / A$ value assuming the PREM density (i.e. $\bar{\rho}=1$ ) and determine its error intervals, from which we can draw the contour lines according to equal values of $n_{e}$ (Fig. 4 (right)).

We used no priors for the fit to remain completely independent of seismological measurements. PREM uncertainties on the outer core density are smaller than a few percent and would therefore heavily constrain the possible combinations of $Z / A$ and $\rho$. In the future, the constraints from absorption tomography on the outer core density could help to narrow down the contour bands. 
Realistic models of the chemical composition of the outer core provide values between 0.466 (iron nickel alloy as in the inner core) and 0.4714 ("exotic" model containing $1 \mathrm{wt} \%{ }^{3} \mathrm{H}$ [15]) and will not be distinguishable with the current generation of neutrino detectors.

The result of oscillation studies presented in the analysis relies on the global best fit of oscillation parameters assuming normal mass ordering [16] and does not include systematic uncertainties. ORCA and other neutrino oscillation experiments will most likely determine the true mass ordering in a shorter time scale than 10 years which is the time scale considered in this simulation and also other oscillation parameters will be determined with a higher precision. An estimation of systematic effects showed that events only traversing the mantle region of the Earth will limit these effects while investigating only the outer core.

\subsection{Summary}

We showed here that KM3NeT, with its two detectors ARCA and ORCA, is an experiment capable of performing both oscillation and absorption tomography of the Earth. Using a Monte Carlo based model to simulate the detector response, we were able to calculate the sensitivities for measurements of the $Z / A$ value of the outer core, which cannot be measured with seismic measurements, as well as of the Earth's density profile. Despite the uncertainties related to neutrino tomography are still much bigger than those provided by geoscience, this new approach to explore the Earth interior can contribute to constrain future models of the planet.

${ }^{3}$ 'Weight percent', meaning one percent of the total mass comes from hydrogen. 


\section{Acknowledgements}

The authors gratefully acknowledge financial support from LabEx UnivEarthS (ANR-10LABX-0023 and ANR-18-IDEX-0001) and from the CNRS Interdisciplinary Program 80PRIME.

\section{References}

[1] A. Donini et al., Neutrino tomography of the Earth, Nature Physics, 15, 37-40, (2019).

[2] M. Dziewonski and D. L. Anderson, Physics of the Earth and Planetary Interiors 25297 (1981).

[3] F. Birch, J. Geophys. Res., 57(2), 227-286 (1952).

[4] S. Adrián-Martinéz et al. "Letter of intent for KM3NeT 2.0". In: Journal of Physics G: Nuclear and Particle Physics (2016).

[5] https://earthref.org/GERMRD/datamodel/ (cit. on pp. 114, 115).

[6] E. Kaminski and M. Javoy, Earth Plan. Sci. Lett. 365 97-107 (2013).

[7] M. Honda et al., Phys. Rev. D 92023004 (2015);

[8] M. Honda et al., Phys. Rev. D 75043006 (2007), [arXiv:astro-ph/0611418]

[9] R. Enberg et al., Phys. Rev. D 78043005 (2008), [arXiv:0806.0418 [astro-ph]].

[10] https://github.com/joaoabcoelho/OscProb

[11] C. Andreopoulos et al., Nucl. Instrum. Meth. A614 87-104 (2010).

[12] S. Bourret et al. PoS ICRC2019 (2020) 1024, DOI: 10.22323/1.358.1024

[13] Simon Bourret. Neutrino oscillations and earth tomography with KM3NeT-ORCA. Physics [physics]. Université Sorbonne Paris Cité, 2018. English. NNT : 2018USPCC247 . tel02491394

[14] M. Hatlo et al. Developments of Mathematical Software Libraries for the LHC experiments, IEEE Trans. Nucl. Sci. 52 (2005).

[15] T. Sakamaki et al., Earth and Planetary Science Letters 287 293-297 (2009).

[16] Esteban, I., Gonzalez-Garcia, M., Maltoni, M. et al. The fate of hints: updated global analysis of three-flavor neutrino oscillations. J. High Energ. Phys. 2020, 178 (2020). https://doi.org/10.1007/JHEP09(2020)178 


\section{Full Author List: KM3NeT Collaboration}

M. Ageron ${ }^{1}$, S. Aiello ${ }^{2}$, A. Albert ${ }^{3,55}$, M. Alshamsi ${ }^{4}$, S. Alves Garre ${ }^{5}$, Z. Aly ${ }^{1}$, A. Ambrosone ${ }^{6,7}$, F. Ameli ${ }^{8}$, M. Andre 9 , G. Androulakis ${ }^{10}$, M. Anghinolfi ${ }^{11}$, M. Anguita ${ }^{12}$, G. Anton ${ }^{13}$, M. Ardid ${ }^{14}$, S. $\operatorname{Ardid}^{14}$, W. Assal ${ }^{1}$, J. Aublin ${ }^{4}$, C. Bagatelas ${ }^{10}$, B. Baret ${ }^{4}$, S. Basegmez du Pree ${ }^{15}$, M. Bendahman ${ }^{4,16}$, F. Benfenati ${ }^{17,18}$, E. Berbee ${ }^{15}$, A. M. van den Berg ${ }^{19}$, V. Bertin ${ }^{1}$, S. Beurthey ${ }^{1}$, V. van Beveren ${ }^{15}$, S. Biagi ${ }^{20}$, M. Billault ${ }^{1}$, M. Bissinger ${ }^{13}$, M. Boettcher ${ }^{21}$, M. Bou Cabo ${ }^{22}$, J. Boumaaza ${ }^{16}$, M. Bouta ${ }^{23}$, C. Boutonnet ${ }^{4}$, G. Bouvet ${ }^{24}$, M. Bouwhuis ${ }^{15}$, C. Bozza ${ }^{25}$, H.Brânzaş ${ }^{26}$, R. Bruijn ${ }^{15,27}$, J. Brunner ${ }^{1}$, R. Bruno ${ }^{2}$, E. Buis ${ }^{28}$, R. Buompane ${ }^{6,29}$, J. Busto ${ }^{1}$, B. Caiffi ${ }^{11}$, L. Caillat ${ }^{1}$, D. Calvo ${ }^{5}$, S. Campion ${ }^{30,8}$, A. Capone ${ }^{30,8}$, H. Carduner ${ }^{24}$, V. Carretero ${ }^{5}$, P. Castaldi ${ }^{17,31}$, S. Celli ${ }^{30,8}$, R. Cereseto ${ }^{11}$, M. Chabab ${ }^{32}$, C. Champion ${ }^{4}$, N. $\mathrm{Chau}^{4}$, A. Chen ${ }^{33}$, S. Cherubinini ${ }^{20,34}$, V. Chiarella ${ }^{35}$, T. Chiarusi ${ }^{17}$, M. Circella ${ }^{36}$, R. Cocimano ${ }^{20}$, J. A. B. Coelho ${ }^{4}$, A. Coleiro ${ }^{4}$, M. Colomer Molla ${ }^{4,5}$, S. Colonges ${ }^{4}$, R. Coniglione ${ }^{20}$, A. Cosquer ${ }^{1}$, P. Coyle ${ }^{1}$, M. Cresta ${ }^{11}$, A. Creusot ${ }^{4}$, A. $\mathrm{Cruz}^{37}$, G. Cuttone ${ }^{20}$, A. D’Amico ${ }^{15}$, R. Dallier ${ }^{24}$, B. De Martino ${ }^{1}$, M. De Palma ${ }^{36,38}$, I. Di Palma ${ }^{30,8}$, A. F. Díaz ${ }^{12}$, D. Diego$\operatorname{Tortosa}^{14}$, C. Distefano ${ }^{20}$, A. Domi ${ }^{15,27}$, C. Donzaud ${ }^{4}$, D. Dornic ${ }^{1}$, M. Dörr ${ }^{39}$, D. Drouhin ${ }^{3,55}$, T. Eberl ${ }^{13}$, A. Eddyamoui ${ }^{16}$, T. van Eeden ${ }^{15}$, D. van Eijk ${ }^{15}$, I. El Bojaddaini ${ }^{23}$, H. Eljarrari ${ }^{16}$, D. Elsaesser ${ }^{39}$, A. Enzenhöfer ${ }^{1}$, V. Espinosa ${ }^{14}$, P. Fermani ${ }^{30,8}$, G. Ferrara ${ }^{20,34}$, M. D. Filipovićc ${ }^{40}$, F. Filippini ${ }^{17,18}$, J. Fransen ${ }^{15}$, L. A. Fusco ${ }^{1}$, D. Gajanana ${ }^{15}$, T. Gal ${ }^{13}$, J. García Méndez ${ }^{14}$, A. Garcia Soto $^{5}$, E. Garçon ${ }^{1}$, F. Garufi ${ }^{6,7}$, C. Gatius ${ }^{15}$, N. Geißelbrecht ${ }^{13}$, L. Gialanella ${ }^{6,29}$, E. Giorgio ${ }^{20}$, S. R. Gozzini ${ }^{5}$, R. Gracia ${ }^{15}$, K. Graf ${ }^{13}$, G. Grella ${ }^{41}$, D. Guderian ${ }^{56}$, C. Guidi ${ }^{11,42}$, B. Guillon ${ }^{43}$, M. Gutiérrez ${ }^{44}$, J. Haefner ${ }^{13}$, S. Hallmann ${ }^{13}$, H. Hamdaoui ${ }^{16}$, H. van Haren ${ }^{45}$, A. Heijboer ${ }^{15}$, A. Hekalo ${ }^{39}$, L. Hennig ${ }^{13}$, S. Henry ${ }^{1}$, J. J. Hernández-Rey ${ }^{5}$, J. Hofestädt ${ }^{13}$, F. Huang ${ }^{1}$, W. Idrissi Ibnsalih ${ }^{6,29}$, A. Ilioni ${ }^{4}$, G. Illuminati ${ }^{17,18,4}$, C. W. James ${ }^{37}$, D. Janezashvili ${ }^{46}$, P. Jansweijer ${ }^{15}$, M. de Jong ${ }^{15,47}$, P. de Jong ${ }^{15,27}$, B. J. Jung ${ }^{15}$, M. Kadler ${ }^{39}$, P. Kalaczyński ${ }^{48}$, O. Kalekin ${ }^{13}$, U. F. Katz ${ }^{13}$, F. Kayzel $^{15}$, P. Keller ${ }^{1}$, N. R. Khan Chowdhury ${ }^{5}$, G. Kistauri ${ }^{46}$, F. van der Knaap ${ }^{28}$, P. Kooijman ${ }^{27,57}$, A. Kouchner ${ }^{4,49}$, M. Kreter ${ }^{21}$, V. Kulikovskiy ${ }^{11}$, M. Labalme ${ }^{43}$, P. Lagier ${ }^{1}$, R. Lahmann ${ }^{13}$, P. Lamare ${ }^{1}$, M. Lamoureux ${ }^{44}$, G. Larosa ${ }^{20}$, C. Lastoria ${ }^{1}$, J. Laurence ${ }^{1}$, A. Lazo $^{5}$, R. Le Breton ${ }^{4}$, E. Le Guirriec ${ }^{1}$, S. Le Stum ${ }^{1}$, G. Lehaut ${ }^{43}$, O. Leonardi ${ }^{20}$, F. Leone ${ }^{20,34}$, E. Leonora ${ }^{2}$, C. Lerouvillois ${ }^{1}$, J. Lesrel ${ }^{4}$, N. Lessing ${ }^{13}$, G. Levi ${ }^{17,18}$, M. Lincetto ${ }^{1}$, M. Lindsey Clark ${ }^{4}$, T. Lipreau ${ }^{24}$, C. LLorens Alvarez ${ }^{14}$, A. Lonardo ${ }^{8}$, F. Longhitano ${ }^{2}$, D. Lopez-Coto ${ }^{44}$, N. Lumb ${ }^{1}$, L. Maderer ${ }^{4}$, J. Majumdar ${ }^{15}$, J. Mańczak ${ }^{5}$, A. Margiotta ${ }^{17,18}$, A. Marinelli ${ }^{6}$, A. Marini ${ }^{1}$, C. Markou $^{10}$, L. Martin ${ }^{24}$, J. A. Martínez-Mora ${ }^{14}$, A. Martini ${ }^{35}$, F. Marzaioli ${ }^{6,29}$,

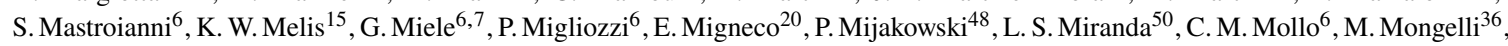
A. Moussa ${ }^{23}$, R. Muller ${ }^{15}$, P. Musico ${ }^{11}$, M. Musumeci ${ }^{20}$, L. Nauta ${ }^{15}$, S. Navas ${ }^{44}$, C. A. Nicolau ${ }^{8}$, B. Nkosi ${ }^{33}$, B. Ó Fearraigh ${ }^{15,27}$, M. O'Sullivan ${ }^{37}$, A. Orlando ${ }^{20}$, G. Ottonello ${ }^{11}$, S. Ottonello ${ }^{11}$, J. Palacios González ${ }^{5}$, G. Papalashviliite, R. Papaleo ${ }^{20}$, C. Pastore ${ }^{36}$, A. M. Păun ${ }^{26}$, G.E. Păvălaşş, G. Pellegrinini ${ }^{17}$, C. Pellegrino ${ }^{18,58}$, M. Perrin-Terrinin ${ }^{1}$, V. Pestel ${ }^{15}$, P. Piattelli ${ }^{20}$, C. Pieterse ${ }^{5}$, O. Pisanti ${ }^{6,7}$, C. Poirè ${ }^{14}$, V. Popa $^{26}$, T. Pradier $^{3}$, F. Pratolongo ${ }^{11}$, I. Probst ${ }^{13}$, G. Pühlhofer ${ }^{51}$, S. Pulvirenti ${ }^{20}$, G. Quéméner ${ }^{43}$, N. Randazzo ${ }^{2}$, A. Rapicavoli ${ }^{34}$, S. Razzaque $^{50}$, D. Real ${ }^{5}$, S. Reck ${ }^{13}$, G. Riccobene ${ }^{20}$, L. Rigalleau ${ }^{24}$, A. Romanov ${ }^{11,42}$, A. Rovelli ${ }^{20}$, J. Royon ${ }^{1}$, F. Salesa Greus ${ }^{5}$, D. F. E. Samtleben ${ }^{15,47}$, A. Sánchez Losa ${ }^{36,5}$, M. Sanguineti ${ }^{11,42}$, A. Santangelo ${ }^{51}$, D. Santonocito ${ }^{20}$, P. Sapienza ${ }^{20}$, J. Schmelling ${ }^{15}$, J. Schnabel ${ }^{13}$, M. F. Schneider ${ }^{13}$, J. Schumann ${ }^{13}$, H. M. Schutte ${ }^{21}$, J. Seneca ${ }^{15}$, I. Sgura ${ }^{36}$, R. Shanidze ${ }^{46}$, A. Sharma ${ }^{52}$, A. Sinopoulou ${ }^{10}$, B. Spisso ${ }^{41,6}$, M. Spurio ${ }^{17,18}$, D. Stavropoulos ${ }^{10}$, J. Steijger ${ }^{15}$, S. M. Stellacci ${ }^{41,6}$, M. Taiuti ${ }^{11,42}$, F. Tatone ${ }^{36}$, Y. Tayalati ${ }^{16}$, E. Tenllado ${ }^{44}$, D. Tézier ${ }^{1}$, T. Thakore ${ }^{5}$, S. Theraube ${ }^{1}$, H. Thiersen $^{21}$, P. Timmer $^{15}$, S. Tingay ${ }^{37}$, S. Tsagkli $^{10}$, V. Tsourapis ${ }^{10}$, E. Tzamariudaki ${ }^{10}$, D. Tzanetatos ${ }^{10}$, C. Valieri ${ }^{17}$, V. Van Elewyck ${ }^{4,49}$, G. Vasileiadis ${ }^{53}$, F. Versari ${ }^{17,18}$, S. Viola $^{20}$, D. Vivolo ${ }^{6,29}$, G. de Wasseige ${ }^{4}$, J. Wilms ${ }^{54}$, R. Wojaczyński ${ }^{48}$, E. de Wolf ${ }^{15,27}$, T. Yousfi ${ }^{23}$, S. Zavatarelli ${ }^{11}$, A. Zegarelli ${ }^{30,8}$, D. Zito ${ }^{20}$, J. D. Zornoza ${ }^{5}$, J. Zúñiga ${ }^{5}$, N. Zywucka ${ }^{21}$.

${ }^{1}$ Aix Marseille Univ, CNRS/IN2P3, CPPM, Marseille, France.

${ }^{2}$ INFN, Sezione di Catania, Via Santa Sofia 64, Catania, 95123 Italy.

${ }^{3}$ Université de Strasbourg, CNRS, IPHC UMR 7178, F-67000 Strasbourg, France.

${ }^{4}$ Université de Paris, CNRS, Astroparticule et Cosmologie, F-75013 Paris, France.

${ }^{5}$ IFIC - Instituto de Física Corpuscular (CSIC - Universitat de València), c/Catedrático José Beltrán, 2, 46980 Paterna, Valencia, Spain. ${ }^{6}$ INFN, Sezione di Napoli, Complesso Universitario di Monte S. Angelo, Via Cintia ed. G, Napoli, 80126 Italy.

${ }^{7}$ Università di Napoli “Federico II", Dip. Scienze Fisiche "E. Pancini”, Complesso Universitario di Monte S. Angelo, Via Cintia ed. G, Napoli, 80126 Italy.

${ }^{8}$ INFN, Sezione di Roma, Piazzale Aldo Moro 2, Roma, 00185 Italy.

${ }^{9}$ Universitat Politècnica de Catalunya, Laboratori d'Aplicacions Bioacústiques, Centre Tecnològic de Vilanova i la Geltrú, Avda. Rambla Exposició, s/n, Vilanova i la Geltrú, 08800 Spain.

${ }^{10}$ NCSR Demokritos, Institute of Nuclear and Particle Physics, Ag. Paraskevi Attikis, Athens, 15310 Greece.

${ }^{11}$ INFN, Sezione di Genova, Via Dodecaneso 33, Genova, 16146 Italy.

${ }^{12}$ University of Granada, Dept. of Computer Architecture and Technology/CITIC, 18071 Granada, Spain.

${ }^{13}$ Friedrich-Alexander-Universität Erlangen-Nürnberg, Erlangen Centre for Astroparticle Physics, Erwin-Rommel-Straße 1, 91058 Erlangen, Germany.

${ }^{14}$ Universitat Politècnica de València, Instituto de Investigación para la Gestión Integrada de las Zonas Costeras, C/ Paranimf, 1, Gandia, 46730 Spain.

${ }^{15}$ Nikhef, National Institute for Subatomic Physics, PO Box 41882, Amsterdam, 1009 DB Netherlands.

${ }^{16}$ University Mohammed V in Rabat, Faculty of Sciences, 4 av. Ibn Battouta, B.P. 1014, R.P. 10000 Rabat, Morocco.

${ }^{17}$ INFN, Sezione di Bologna, v.le C. Berti-Pichat, 6/2, Bologna, 40127 Italy.

${ }^{4}$ also at Dipartimento di Fisica, INFN Sezione di Padova and Università di Padova, I-35131, Padova, Italy 
${ }^{18}$ Università di Bologna, Dipartimento di Fisica e Astronomia, v.le C. Berti-Pichat, 6/2, Bologna, 40127 Italy.

${ }^{19}$ KVI-CART University of Groningen, Groningen, the Netherlands.

${ }^{20}$ INFN, Laboratori Nazionali del Sud, Via S. Sofia 62, Catania, 95123 Italy.

${ }^{21}$ North-West University, Centre for Space Research, Private Bag X6001, Potchefstroom, 2520 South Africa.

${ }^{22}$ Instituto Español de Oceanografía, Unidad Mixta IEO-UPV, C/ Paranimf, 1, Gandia, 46730 Spain.

${ }^{23}$ University Mohammed I, Faculty of Sciences, BV Mohammed VI, B.P. 717, R.P. 60000 Oujda, Morocco.

${ }^{24}$ Subatech, IMT Atlantique, IN2P3-CNRS, Université de Nantes, 4 rue Alfred Kastler - La Chantrerie, Nantes, BP 2072244307 France.

${ }^{25}$ Università di Salerno e INFN Gruppo Collegato di Salerno, Dipartimento di Matematica, Via Giovanni Paolo II 132, Fisciano, 84084 Italy.

${ }^{26}$ ISS, Atomistilor 409, Măgurele, RO-077125 Romania.

${ }^{27}$ University of Amsterdam, Institute of Physics/IHEF, PO Box 94216, Amsterdam, 1090 GE Netherlands.

${ }^{28}$ TNO, Technical Sciences, PO Box 155, Delft, 2600 AD Netherlands.

${ }^{29}$ Università degli Studi della Campania "Luigi Vanvitelli", Dipartimento di Matematica e Fisica, viale Lincoln 5, Caserta, 81100 Italy.

${ }^{30}$ Università La Sapienza, Dipartimento di Fisica, Piazzale Aldo Moro 2, Roma, 00185 Italy.

${ }^{31}$ Università di Bologna, Dipartimento di Ingegneria dell'Energia Elettrica e dell'Informazione "Guglielmo Marconi", Via dell'Università 50, Cesena, 47521 Italia.

${ }^{32}$ Cadi Ayyad University, Physics Department, Faculty of Science Semlalia, Av. My Abdellah, P.O.B. 2390, Marrakech, 40000 Morocco.

${ }^{33}$ University of the Witwatersrand, School of Physics, Private Bag 3, Johannesburg, Wits 2050 South Africa.

${ }^{34}$ Università di Catania, Dipartimento di Fisica e Astronomia "Ettore Majorana", Via Santa Sofia 64, Catania, 95123 Italy.

${ }^{35}$ INFN, LNF, Via Enrico Fermi, 40, Frascati, 00044 Italy.

${ }^{36}$ INFN, Sezione di Bari, via Orabona, 4, Bari, 70125 Italy.

${ }^{37}$ International Centre for Radio Astronomy Research, Curtin University, Bentley, WA 6102, Australia.

${ }^{38}$ University of Bari, Via Amendola 173, Bari, 70126 Italy.

${ }^{39}$ University Würzburg, Emil-Fischer-Straße 31, Würzburg, 97074 Germany.

${ }^{40}$ Western Sydney University, School of Computing, Engineering and Mathematics, Locked Bag 1797, Penrith, NSW 2751 Australia.

${ }^{41}$ Università di Salerno e INFN Gruppo Collegato di Salerno, Dipartimento di Fisica, Via Giovanni Paolo II 132, Fisciano, 84084 Italy.

${ }^{42}$ Università di Genova, Via Dodecaneso 33, Genova, 16146 Italy.

${ }^{43}$ Normandie Univ, ENSICAEN, UNICAEN, CNRS/IN2P3, LPC Caen, LPCCAEN, 6 boulevard Maréchal Juin, Caen, 14050 France.

${ }^{44}$ University of Granada, Dpto. de Física Teórica y del Cosmos \& C.A.F.P.E., 18071 Granada, Spain.

${ }^{45}$ NIOZ (Royal Netherlands Institute for Sea Research), PO Box 59, Den Burg, Texel, 1790 AB, the Netherlands.

${ }^{46}$ Tbilisi State University, Department of Physics, 3, Chavchavadze Ave., Tbilisi, 0179 Georgia.

${ }^{47}$ Leiden University, Leiden Institute of Physics, PO Box 9504, Leiden, 2300 RA Netherlands.

${ }^{48}$ National Centre for Nuclear Research, 02-093 Warsaw, Poland.

${ }^{49}$ Institut Universitaire de France, 1 rue Descartes, Paris, 75005 France.

${ }^{50}$ University of Johannesburg, Department Physics, PO Box 524, Auckland Park, 2006 South Africa.

${ }^{51}$ Eberhard Karls Universität Tübingen, Institut für Astronomie und Astrophysik, Sand 1, Tübingen, 72076 Germany.

${ }^{52}$ Università di Pisa, Dipartimento di Fisica, Largo Bruno Pontecorvo 3, Pisa, 56127 Italy.

${ }^{53}$ Laboratoire Univers et Particules de Montpellier, Place Eugène Bataillon - CC 72, Montpellier Cédex 05, 34095 France.

${ }^{54}$ Friedrich-Alexander-Universität Erlangen-Nürnberg, Remeis Sternwarte, Sternwartstraße 7, 96049 Bamberg, Germany.

${ }^{55}$ Université de Haute Alsace, 68100 Mulhouse Cedex, France.

${ }^{56}$ University of Münster, Institut für Kernphysik, Wilhelm-Klemm-Str. 9, Münster, 48149 Germany.

${ }^{57}$ Utrecht University, Department of Physics and Astronomy, PO Box 80000, Utrecht, 3508 TA Netherlands.

${ }^{58}$ INFN, CNAF, v.le C. Berti-Pichat, 6/2, Bologna, 40127 Italy. 